\title{
ESPECTROMETRÍA DE IMAGEN PARA EL ESTUDIO Y CARACTERIZACIÓN DE LA CONTAMINACIÓN POR METALES PESADOS
}

\author{
IMAGE SPECTROMETRY FOR THE STUDY AND CHARACTERIZATION OF CONTAMINATION BY HEAVY METALS \\ Francisco Cabrera-Torres ${ }^{\mathrm{a}, \mathrm{b},{ }^{*},}$, Mercedes Farjas-Abadíaa, Juan Gregorio Rejas-Ayugaa ${ }^{\mathrm{c}, \mathrm{d}}$ \\ a Departamento de Ingeniería Topográfica y Cartografía, Universidad Politécnica de Madrid, 28031 Madrid, España. \\ fd.cabrera@alumnos.upm.es; m.farjas@upm.es \\ b Instituto Geográfico Militar, IGM, 170413 Quito, Ecuador. franchesco_c7@hotmail.com \\ c Departamento de Ingeniería y Morfología del Terreno, Universidad Politécnica de Madrid, 28040 Madrid, España. \\ juangregorio.rejas@upm.es \\ d Instituto Nacional de Técnica Aeroespacial, INTA, 28850 Torrejón de Ardoz, España. rejasaj@inta.es
}

\begin{abstract}
:
Environmental degradation or contamination is subject to the presence of heavy metals such as: arsenic, cadmium, mercury, nickel, lead, etc., which may have a geogenic (natural) or anthropogenic origin (produced by hazardous waste), the latter bring with them negative consequences for human health, due to the bioaccumulation of trace elements. Today, spectrometric techniques, through the integration of geospatial technologies and multi-source data, are considered an alternative to traditional field sampling methods, and their use is gaining strength every day. This article uses the so-called "Crosta technique" to detect the presence of characteristic minerals existing in the Southeast Regional Park of the Autonomous Community of Madrid, and, on the other hand, to locate the existence of heavy metals. In a qualitative evaluation, applying the "Crosta composition", a high concordance between the minerals bentonite and sepiolite is evidenced with the official contrast cartography, from the Geological and Mining Institute of Spain, in addition, a slight modification was made in the input bands of the "Crosta composition", obtaining an overestimation of the minerals. Finally, by means of a visual interpretation of those main components that contain the spectral information related to heavy metals, a list of coordinates was generated, which will be validated in field campaigns, where the use of spectroradiometers and the chemical analysis of samples will be key to determine the goodness of the results.
\end{abstract}

Key words: remote sensing, heavy metals, imaging spectrometry, geospatial technologies, spectral characterization, advanced geomatics

\section{Resumen:}

La degradación o contaminación ambiental se encuentra sujeta a la presencia de metales pesados como: arsénico, cadmio, mercurio, níquel, plomo, etc., los cuales pueden tener origen geogénico (natural) o antropogénico (producidos por residuos peligrosos), estos últimos, traen consigo consecuencias negativas para la salud humana, debido a la bioacumulación de elementos traza. Hoy en día, las técnicas espectrométricas, mediante la integración de tecnologías geoespaciales y datos multifuente, son consideradas como una alternativa a los métodos tradicionales de muestreo de campo, y su empleo cada día cobra más fuerza. El presente artículo, emplea la denominada "técnica de Crosta" para detectar la presencia de minerales característicos existentes en el Parque Regional del Sureste de la Comunidad Autónoma de Madrid, y, por otro lado, para localizar la existencia de metales pesados. De manera cualitativa, aplicando la "composición Crosta", se evidencia una alta concordancia entre los minerales bentonita y sepiolita con la cartografía oficial de contraste, procedente del Instituto Geológico y Minero de España, además, se realizó una leve modificación en las bandas de entrada de la "composición Crosta", obteniendo una sobre estimación de los minerales. Por último, mediante una interpretación visual de aquellas componentes principales que contienen la información espectral relativa a metales pesados, se generó un listado de coordenadas, las cuales serán validadas en campañas de campo, en donde el uso de espectroradiómetros y el análisis químico de muestras serán claves para determinar la bondad de los resultados.

Palabras clave: teledetección, metales pesados, espectrometría de imagen, tecnologías geoespaciales, caracterización espectral, geomática avanzada

\section{Introducción}

Los metales pesados se caracterizan por presentar una densidad mayor que $4 \mathrm{~g} / \mathrm{cm}^{3}$ y son considerados contaminantes prioritarios debido a su no degradabilidad y su larga persistencia en el ambiente. Su origen puede ser geogénico, procedentes de la meteorización de la roca madre, actividad volcánica o lixiviado de mineralizaciones, o de origen antropogénico, producto de

‘Corresponding Author: Francisco Cabrera-Torres, franchesco_c7@hotmail.com 
residuos peligrosos derivados de actividades: industriales, mineras, agrícolas, etc., y de residuos sólidos urbanos. Algunos elementos son transportados y distribuidos a los ecosistemas, e incorporados en la cadena trófica (Durães et al. 2017; Galán and Romero 2008; Londoño et al. 2016).

La falta de control de emisiones de estos residuos puede traer consigo consecuencias negativas para la salud humana, debido a la bioacumulación de elementos traza, originada por su incremento dentro del sistema alimenticio o por el consumo en bajas cantidades en un largo período de tiempo, trayendo como consecuencia enfermedades crónicas, incapacidad o la muerte (Siegel 2002). Las primeras evidencias de personas afectadas se encontraron en Japón, debido a la contaminación de metilmercurio en los años 50 y de Cd en los 60 , además, en la India, entre los años 80 y 90, el As generó un estado de alarma entre sus pobladores (Adriano 2001).

De manera similar, la presencia de elementos traza en el suelo genera una modificación negativa denominada degradación o contaminación, y en la vegetación genera estrés, ocasionando cambios metabólicos y morfológicos considerables (Arellano et al. 2015; Davids and Tyler 2003; Galán and Romero 2008).

Hoy en día, una alternativa a los métodos tradicionales de muestreo de campo, es a través de la espectrometría, mediante el uso de imágenes (satelitales 0 aerotransportadas) o espectroradiómetros, la cual permite identificar longitudes de onda diagnóstico de los metales pesados, así como diferencias de reflectancias por parte de los diferentes objetivos afectados por estos elementos (Arellano et al. 2015; Rosso et al. 2005). Shin et al. (2020) consideran a la espectrometría como no destructiva, rentable, repetible y como un método efectivo a la hora de determinar concentraciones de metales pesados en suelos.

Esta técnica tiene un amplio beneficio para estudios de agricultura de precisión, permitiendo a los agricultores refinar la fertilización, evaluar el estado nutricional de los cultivos, controlar la irrigación y/o detectar cambios fisiológicos en las especies vegetales por plagas o enfermedades, a partir de índices de vegetación, útiles para la determinación de parámetros agroforestales (actividad fotosintética, estrés hídrico, nivel de productividad, etc.) (Meza et al. 2020; Soca et al. 2020).

Varias técnicas espectrométricas son empleadas para la exploración e identificación de bandas sensibles a diferentes elementos químicos, por ejemplo, índices espectrales de diferencia normalizada (Das et al. 2020; Yao et al. 2010), y análisis de componentes principales (ACP) mediante "técnica de Crosta" (Rejas et al. 2014; Bragado et al. 2009).

Haciendo hincapié en la "técnica de Crosta", la misma fue desarrollada por Crosta y Moore en el año de 1989, y perfeccionada por Loughlin, en 1991, es considerada como una técnica rápida, robusta y fiable, la cual se basa en la transformación de componentes principales (CP) y en el análisis de las cargas factoriales de los vectores propios (Loughlin 1991).

La reducción en la entrada del número de bandas de la imagen, propuesta por Loughlin (1991), aumenta la probabilidad de que los objetivos o materiales de interés puedan ser mapeados. De manera específica, esta técnica parte de la selección de bandas o longitudes de onda, las cuales presentan rasgos distintivos de absortividad y reflectividad (Crósta et al. 2003; Bragado et al. 2009).

En función de lo descrito anteriormente, el objetivo del presente artículo es estudiar y caracterizar los minerales que contienen metales pesados mediante el empleo de la "técnica de Crosta", haciendo uso de imágenes procedentes del programa Copernicus, por ende, en los capítulos subsiguientes se describe el diseño experimental en base al estado de situación actual de la investigación.

\section{Zona de estudio}

La zona de estudio seleccionada corresponde al Parque Regional del Sureste (PRS) de la Comunidad Autónoma de Madrid (Fig. 1), debido a varios factores, uno de ellos corresponde a la existencia de un elevado número de canteras, además, existen reportes en prensa y foros, de diferentes fechas, en donde resaltan la contaminación que sufre el PRS (Castillejo 2019; Fonseca 2016), por último, la disponibilidad de geoinformación existente (cartografía temática, imágenes satelitales, etc.).

EI PRS posee una superficie de $314,72 \mathrm{~km}^{2}$, extendiéndose de norte a sur, desde San Fernando de Henares hasta Ciempozuelos. En cuanto al aspecto físico se pueden diferenciar claramente dos unidades ambientales, la primera asociada a los relieves colinados terciarios, donde las zonas menores a $650 \mathrm{msnm}$ presentan litologías asociadas a yesos, margas, sepiolita y arenas micáceas, y las superiores presentan calizas dolomíticas y sílex, y la segunda unidad hace referencia a los valles y terrazas aluviales, compuestos principalmente de gravas, arenas y arcillas (IGME 1998).

En cuanto a la cobertura vegetal priman los cultivos herbáceos sobre los valles o terrazas aluviales, al oeste del río Jarama se los encuentra asociados con pastizales y matorrales, y en los relieves colinados terciarios ubicados al sur del río Tajuña y al este de Arganda del Rey se asocian con cultivos como olivares y viñedos. A lo largo de toda la zona de estudio es característico encontrar áreas de extracción (canteras) y vertederos (IGN 2011).

En el aspecto fluvial se evidencia que los ríos Manzanares (atraviesa la ciudad de Madrid de noroeste a sureste), Henares, Tajuña y Tajo desembocan en el río Jarama $\left(\sim 30 \mathrm{~m}^{3} / \mathrm{s}\right)$, siendo el más representativo de esta unidad, ya que recorre longitudinalmente el PRS.

\section{Caracterización y transformación de imagen}

\subsection{Materiales}

Para la realización de esta fase del estudio se empleó geoinformación de acceso libre (Tabla 1), proveniente de organismos oficiales, la misma fue estandarizada bajo un mismo sistema de referencia (WGS84 UTM Z30N) y enmarcada en función del límite del PRS. 


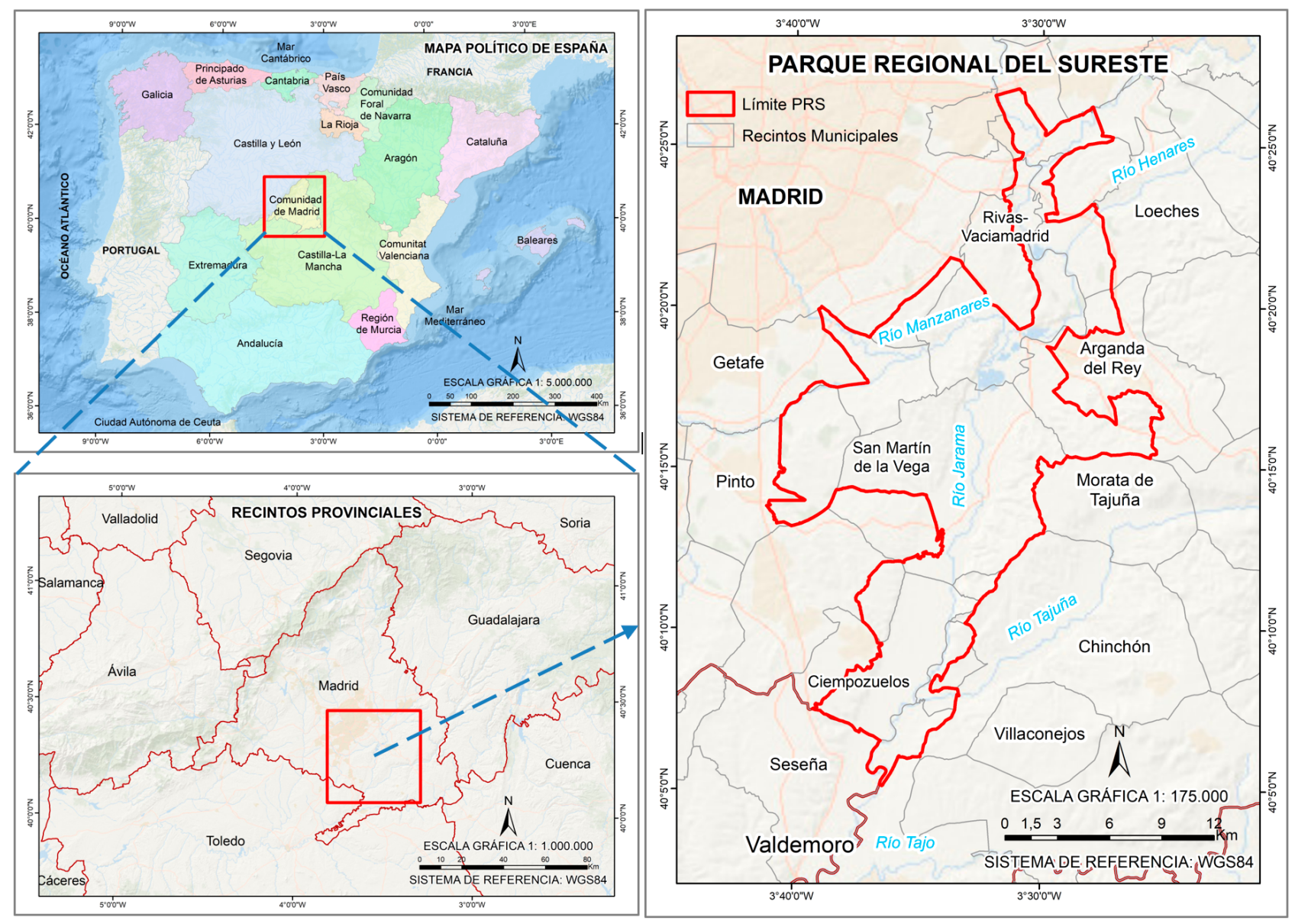

Figura 1: Localización del Parque Regional del Sureste. Fuente cartográfica: (IGN 2019; CM 2019).

Tabla 1: Fuentes de geoinformación. *Bottom of Atmosphere reflectance images ( $\rho \mathrm{BOA})$. ${ }^{*}$ Sistema de Información sobre Ocupación del Suelo de España (SIOSE).

\begin{tabular}{|c|c|c|}
\hline Cobertura & Organismo & Descripción \\
\hline Imágenes Sentinel-2 & European Space Agency (ESA) & $\begin{array}{c}\text { Fecha: 03/01/2021; Formato: .jp2; } \\
\text { Tipo: } L 2 A\left(\rho \mathrm{BOA}^{*}\right)\end{array}$ \\
\hline Límites administrativos & Instituto Geográfico Nacional (IGN) & Fecha: 01/02/2019; Formato: .shp \\
\hline Límite del PRS & Datos abiertos de la Comunidad de Madrid (CM) & Fecha: 18/03/2019; Formato: .shp \\
\hline $\begin{array}{l}\text { Mapa geológico, metalogenético, y } \\
\text { rx industriales }\end{array}$ & Instituto Geológico y Minero de España (IGME) & $\begin{array}{l}\text { Años: } 1972 \text { - 2003; Formato: .jpg; } \\
\text { Esc: } 50,200 \text { y } 400 k\end{array}$ \\
\hline SIOSE $^{* *}$ & IGN & Año: 2011; Formato: .shp; Esc: 25k \\
\hline Banco de firmas espectrales & $\begin{array}{c}\text { Jet Propulsion Laboratory (JPL) y United States } \\
\text { Geological Survey (USGS) }\end{array}$ & $\begin{array}{l}\text { Formato: .asc; Longitud de onda: } 0.4 \mathrm{a} \\
\qquad 2.5 \mu \mathrm{m}\end{array}$ \\
\hline
\end{tabular}

\subsection{Técnica de Crosta}

Los minerales con mayor abundancia en el PRS son la bentonita (compuesta por $\sim 92 \%$ de montmorillonita) y la sepiolita (de la familia de los filosilicatos) en base a las cartas geológicas del IGME (1998), los cuales fueron seleccionados para demostrar el flujo de procesos de la técnica en estudio. Para esto, preliminarmente se extrae del banco de firmas espectrales (Fig. 2), dos longitudes de onda absortivas y dos reflectivas de cada mineral, dichas longitudes deben ser análogas a las bandas en las que opera el Sentinel-2, como se expone en la Tabla 2.

Cabe mencionar que, las bandas 11 (reflexión) y 12 (absorción) exhiben el mayor contraste espectral en ambos casos, esto permitirá hallar la CP adecuada derivada de la transformación lineal, denominándose imagen B para la bentonita e imagen $\mathrm{S}$ para el caso de la sepiolita. Dicho hallazgo se sustenta en el análisis de los signos y cargas factoriales presentes en la matriz de vectores propios. Se debe considerar que, la CP1 corresponde al brillo de la escena, la CP2 describe el contraste entre grupo de bandas, por ende, el análisis per se recae sobre la $\mathrm{CP} 3$ y $\mathrm{CP} 4$, seleccionando preferencialmente aquella PC con cargas fuertes y signos opuestos (Loughlin 1991; Rejas et al. 2014).

Una vez seleccionadas las $C P$, se vincula al canal rojo la imagen $\mathrm{B}$, al canal azul la imagen $\mathrm{S}$, y para el canal verde se procede a calcular la imagen mediante la operación 
$(B x S)$, la cual presenta una leve modificación a la "composición Crosta" original. Los resultados fueron presentados visualmente en el plano RGB, siendo esta una manera usual de hacerlo. Cabe mencionar que, la modificación implementada tiene por finalidad resaltar características o elementos específicos en la zona de estudio mediante el empleo de nuevos algoritmos.

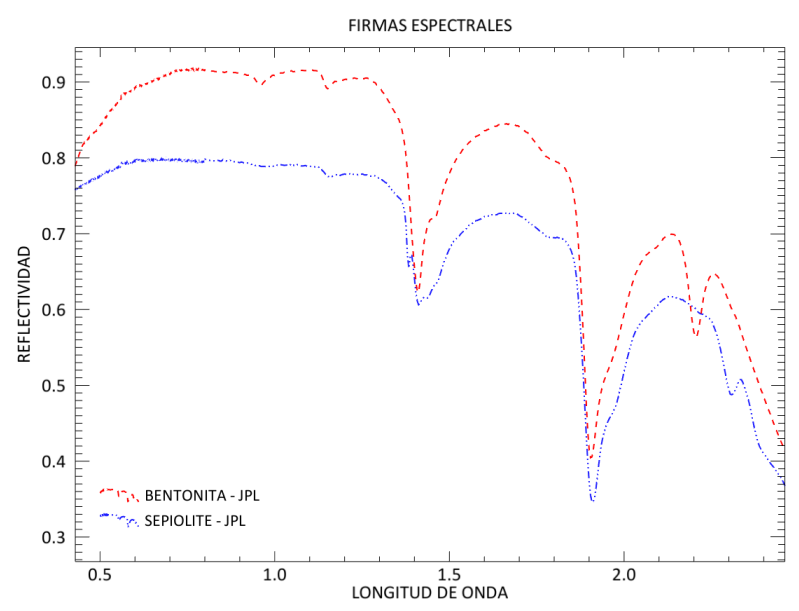

Figura 2: Firmas espectrales tipo. Fuente: (JPL 2018).

Tabla 2: Bandas absortivas y reflectivas de Sentinel-2 empleadas en el ACP para la detección de bentonita y sepiolita.

\begin{tabular}{c|c|c} 
Mineral & Absortividad & Reflectividad \\
\hline Bentonita & B9 $(945 \mathrm{~nm})$ & B7 $(783 \mathrm{~nm})$ \\
& B12 $(2190 \mathrm{~nm})$ & B11 $(1610 \mathrm{~nm})$ \\
Sepiolita & B3 $(560 \mathrm{~nm})$ & B5 $(705 \mathrm{~nm})$ \\
& B12 $(2190 \mathrm{~nm})$ & B11 $(1610 \mathrm{~nm})$
\end{tabular}

Todo el proceso descrito en este apartado fue aplicado para la detección de los siguientes metales pesados: As, $\mathrm{Cd}, \mathrm{Cr}, \mathrm{Cu}, \mathrm{Hg}, \mathrm{Ni}, \mathrm{Pb}$ y $\mathrm{Zn}$, los cuales se consideran como muy tóxicos, fácilmente disponibles y fácilmente movilizados por la actividad humana (Galán and Romero 2008). Para esto se analizó la importancia geológicaeconómica con la finalidad de seleccionar minerales que contienen estos elementos traza y presenten rasgos distintivos de absortividad y reflectividad.

\section{Discusión de resultados}

Los resultados obtenidos fueron contrastados con información geológica, metalogenético, y de minerales y rocas industriales generados por el IGME, esto en cuanto a los minerales de mayor abundancia. Para el caso de los minerales con alto contenido de metales pesados, se generó una tabla, la cual contiene coordenadas de aquellas localidades con supuesta presencia de estos elementos traza, la misma que será verificada a través de campañas de campo.

\subsection{Minerales característicos del PRS}

\subsubsection{Técnica de Crosta}

Como se mencionó anteriormente el análisis de las cargas factoriales y de sus signos, dentro de la matriz de vectores propios, es fundamental para seleccionar la CP que contiene la información espectral relativa a la bentonita y sepiolita, como se indica en las Tablas 3 y 4 .

Tabla 3: Matriz de vectores y valores propios - Bentonita.

\begin{tabular}{c|c|c|c|c|c} 
2021_PRS & $B 7$ & $B 9$ & $B 11$ & $B 12$ & $\begin{array}{c}\text { Valor } \\
\text { propio } \\
\text { (\%) }\end{array}$ \\
\hline CP1 & -0.48 & -0.37 & -0.61 & -0.51 & 78.92 \\
CP2 & -0.54 & -0.57 & 0.35 & 0.51 & 17.04 \\
CP3 & -0.69 & 0.72 & 0.08 & 0.02 & 3.14 \\
CP4 & 0.06 & 0.12 & -0.71 & 0.69 & 0.90
\end{tabular}

Tabla 4: Matriz de vectores y valores propios - Sepiolita

\begin{tabular}{c|c|c|c|c|c} 
2021_PRS & $B 7$ & $B 9$ & $B 11$ & $B 12$ & $\begin{array}{c}\text { Valor } \\
\text { propio } \\
\text { (\%) }\end{array}$ \\
\hline CP1 & 0.35 & 0.47 & 0.60 & 0.54 & 89.11 \\
CP2 & 0.70 & 0.44 & -0.45 & -0.33 & 8.42 \\
CP3 & 0.31 & -0.44 & -0.47 & 0.70 & 1.49 \\
CP4 & 0.54 & -0.63 & 0.46 & -0.32 & 0.99
\end{tabular}

En el caso de la bentonita (imagen B) se seleccionó la CP4 debido a que presenta fuertes cargas factoriales y de signos opuestos sobre las bandas 11 y 12, las cuales exhiben el mayor contraste espectral, debido a la presencia de $\mathrm{AlOH}$ y de contenido de agua en su estructura. Cabe mencionar que, la bentonita es una arcilla de grano muy fino, por ende, su presencia se manifiesta sobre todo en aquellas porciones del espectro que van de los 1100 a los $2500 \mathrm{~nm}$ (Shin et al. 2020). Posteriormente, se realizó una negación de la CP4 con la finalidad de que el mineral pueda ser resaltado por píxeles brillantes, en lugar de píxeles oscuros.

En cuanto a la sepiolita (imagen S), también perteneciente a la familia de los filosilicatos, el mayor contraste espectral, de igual forma, se encuentra en las bandas 11 y 12, por ende, se seleccionó la CP3, a la cual se le aplicó también una negación.

\subsubsection{Composición Crosta original y modificada}

La Figura 3 muestra los resultados obtenidos de las composiciones, evidenciándose en la Figura 3a que la bentonita es representada con colores naranja y café oscuro, la sepiolita con color azul claro, las zonas urbanas con color negro (ausencia de estos minerales), y aquellas zonas de color cian tenue corresponde a una mezcla de sepiolita y bentonita, predominando el primero.

Por otro lado, en la Figura $3 \mathrm{~b}$ se evidencia una sobre estimación de minerales en lugares específicos, originado por el factor multiplicativo. Se distingue de manera general tres colores, el verde que se ubica sobre las zonas urbanas que correspondería en teoría a una mezcla entre bentonita y sepiolita, el color magenta pertenecería de igual forma a zonas con mixtura de ambos minerales, y el color violeta resalta aquellos lugares con mayor presencia de sepiolita.

De esta forma se constata que la "composición Crosta" original presenta una mayor concordancia con la información temática de contraste, sin embargo, no se 
logra apreciar de una forma clara, aquellas zonas donde existen una mixtura de minerales. Por otro lado, el producto derivado de la modificación realizada, permite una clara detección del mineral sepiolita.

Adicionalmente, se presenta en la Figura 4, ejemplos puntuales de la "composición Crosta" original, contrastados con las menas del mapa metalogenético del IGME, en el cual se encuentran cartografiadas dieciséis localidades con presencia de los minerales en estudio.
En la Figura 4a se puede evidenciar la presencia de bentonita (color café oscuro), localizándose las zonas 1 y 2 junto a la desembocadura del río Manzanares en el Jarama. Por otro lado, en la Figura $4 \mathrm{c}$ se denota la presencia del mineral sepiolita, localizado en los alrededores de Vicálvaro (zonas 3 y 4). Se adjunta la Tabla 5 que contiene las coordenadas de las zonas previamente indicadas.

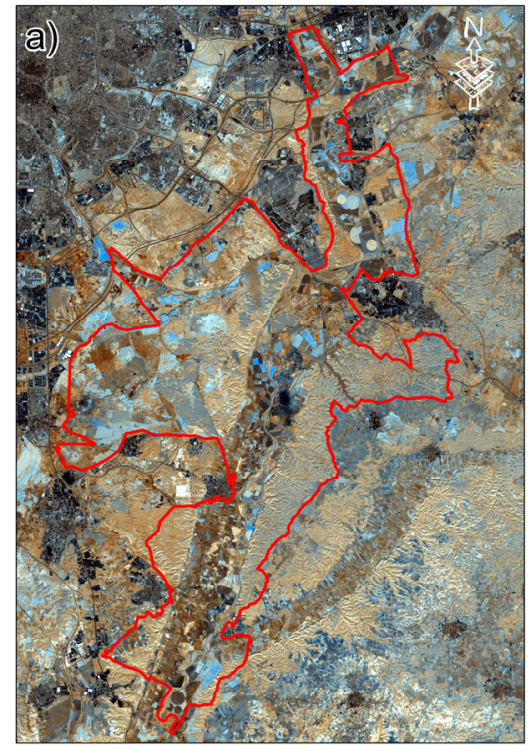

(a)

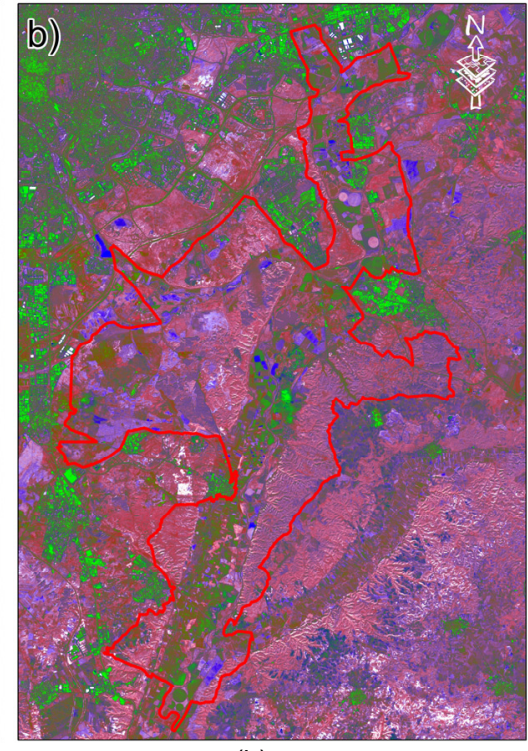

(b)

Figura 3: Composición Crosta: a) Original; b) Modificada.

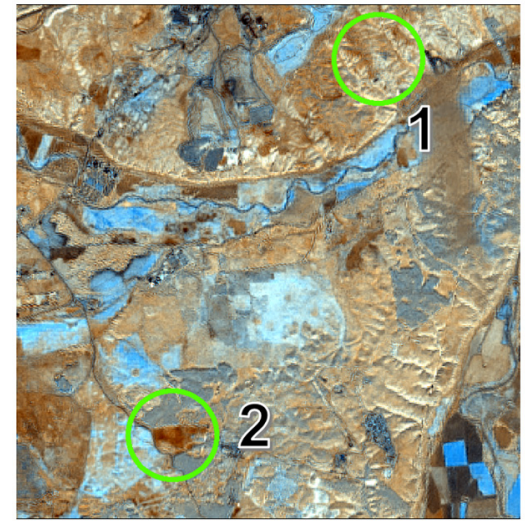

(a)

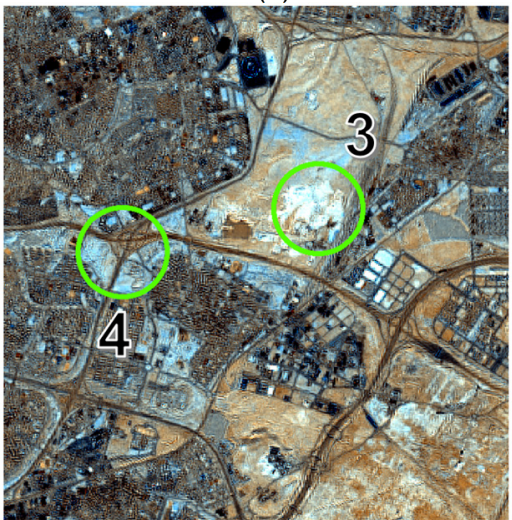

(c)

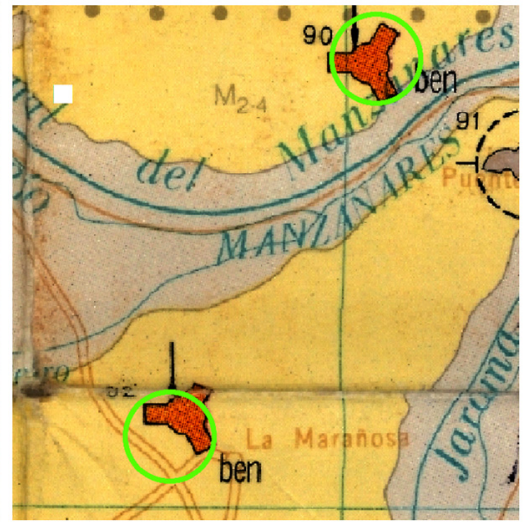

(b)

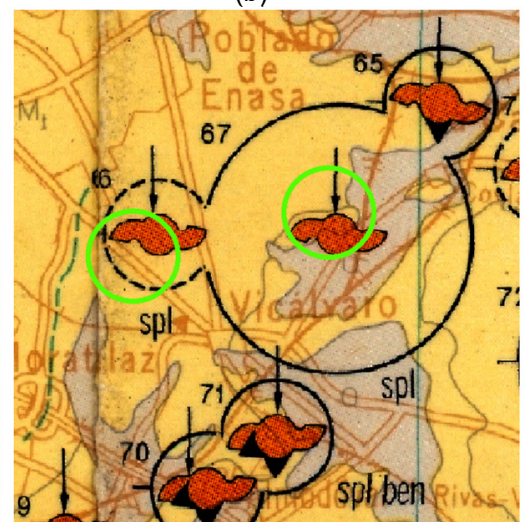

(d)

Figura 4: Ejemplos puntuales de la composición Crostra: a) Detección bentonita, Imagen Sentinel-2; b) Mapa metalogenético de a); c) Detección sepiolita, Imagen Sentinel-2; d) Mapa metalogenético de c). 
Es de importancia mencionar que, debido a la estacionalidad y a las bandas empleadas en el ACP podría existir una variablidad en los resultados obtenidos. Como se mencionó anteriormente, el presente estudio parte de una imagen Sentinel-2, la cual corresponde a la época de invierno (03/01/2021), sin embargo, para el caso puntual de la bentonita, se trabajó con una imagen adicional de las mismas características, correspondiente a la época de verano (12/08/2018), en la cual se detectó una menor presencia de dicho minetal a lo largo de la zona de estudio, sobre todo al este de Rivas Vaciamadrid y en la periferia de Torrejón de Ardoz.

Tabla 5: Coordenadas de zonas contrastadas (WGS84 UTM $\mathrm{Z30N})$.

\begin{tabular}{c|c|c} 
Número & $X(m)$ & $Y(m)$ \\
\hline 1 & 451823 & 4464340 \\
2 & 448886 & 4458863 \\
3 & 450140 & 4474203 \\
4 & 446933 & 4473553
\end{tabular}

\subsection{Minerales con presencia de metales pesados}

De manera similar, la "técnica de Crosta" fue empleada para la obtención de imágenes que manifiesten la presencia, o no, de metales pesados. En la Tabla 6 se detallan las bandas Sentinel-2 con mayor contraste espectral, empleadas en el ACP.

Tabla 6: Bandas absortivas y reflectivas de Sentinel-2 empleadas en el ACP para la detección de elementos traza.

\begin{tabular}{|c|c|c|}
\hline Metal & Bandas absortivas & Bandas reflectivas \\
\hline As & 1 y 8 & 5 y 12 \\
\hline $\mathrm{Cd}$ & 4 y 8 & 7 y 12 \\
\hline $\mathrm{Cr}$ & 4 y 12 & 3 y $8 A$ \\
\hline $\mathrm{Cu}$ & 4 y 11 & 6 y 12 \\
\hline $\mathrm{Hg}$ & $8 \mathrm{~A}$ y 12 & 7 y 11 \\
\hline $\mathrm{Ni}$ & $8 \mathrm{~A}$ y 11 & 9 y 12 \\
\hline $\mathrm{Pb}$ & 3 y 6 & 5 y 12 \\
\hline $\mathrm{Zn}$ & 6 y 12 & 4 y 11 \\
\hline
\end{tabular}

Adicionalmente, mediante una interpretación visual apoyada en imágenes de alta resolución y mapas temáticos procedentes de organismos oficiales, se identicaron sitios con supuesta presencia de elementos traza, mismos que serán verificados en campañas de campo, con la finalidad de evaluar la bondad que tiene la "técnica de Crosta" para aislar metales pesados mediante imágenes Sentinel-2. Las coordenadas de dichos sitios y su respectiva espacialización se encuentran plasmadas en la Tabla 7 y en la Figura 5 respectivamente.

\section{Conclusiones y acciones a corto plazo}

Se corrobora que la "técnica de Crosta" es de amplia utilidad para trabajar en la detección de minerales, obteniendo buenos resultados cualitativos al momento de contrastar los productos derivados con la geoinformación del organismo rector en geología dentro de España.
Previo a la aplicación de la multiplicación en la "composición Crosta" modificada sería conveniente normalizar los datos de entrada.

Tabla 7: Puntos representativos con posible presencia de metales pesados (WGS84 UTM Z30N).

\begin{tabular}{c|c|c|c|c}
$l d$ & $X(m)$ & $Y(m)$ & Elementos & Uso \\
\hline 1 & 444221 & 4464909 & $\mathrm{Cd}, \mathrm{Cu}, \mathrm{Ni}$ & Cultivo \\
2 & 460144 & 4464973 & $\mathrm{As}, \mathrm{Pb}$ & Cultivo \\
3 & 456572 & 4470704 & $\mathrm{Cu}, \mathrm{Ni}$ & Cantera \\
4 & 467272 & 4459416 & $\mathrm{As}, \mathrm{Hg}, \mathrm{Pb}$ & Cantera \\
5 & 464145 & 4454701 & $\mathrm{As}, \mathrm{Hg}, \mathrm{Ni}, \mathrm{Pb}$ & Cantera \\
6 & 454143 & 4456226 & $\mathrm{Cr}, \mathrm{Cu}, \mathrm{Ni}$ & Agua \\
7 & 443936 & 4455146 & $\mathrm{Cu}, \mathrm{Ni}$ & Pastizal \\
8 & 438205 & 4459988 & $\mathrm{Cd}, \mathrm{Cr}, \mathrm{Cu}$ & Suelo \\
9 & 448762 & 4450987 & $\mathrm{Hg}, \mathrm{Pb}, \mathrm{Zn}$ & Cantera \\
10 & 452699 & 4449002 & $\mathrm{Cu}, \mathrm{Ni}$ & Cultivo \\
11 & 463074 & 4433043 & $\mathrm{As}, \mathrm{Pb}, \mathrm{Zn}$ & Cultivo \\
12 & 468687 & 4433275 & $\mathrm{As}, \mathrm{Cd}, \mathrm{Hg}$ & Pastizal \\
13 & 463487 & 4477754 & $\mathrm{As}, \mathrm{Hg}, \mathrm{Pb}$ & Cantera \\
14 & 461965 & 448683 & $\mathrm{Hg}, \mathrm{Zn}$ & Suelo \\
15 & 462019 & 4448352 & $\mathrm{Cu}, \mathrm{Ni}$ & Olivar \\
16 & 454346 & 4461047 & $\mathrm{As}, \mathrm{Pb}$ & Agua \\
& & & &
\end{tabular}

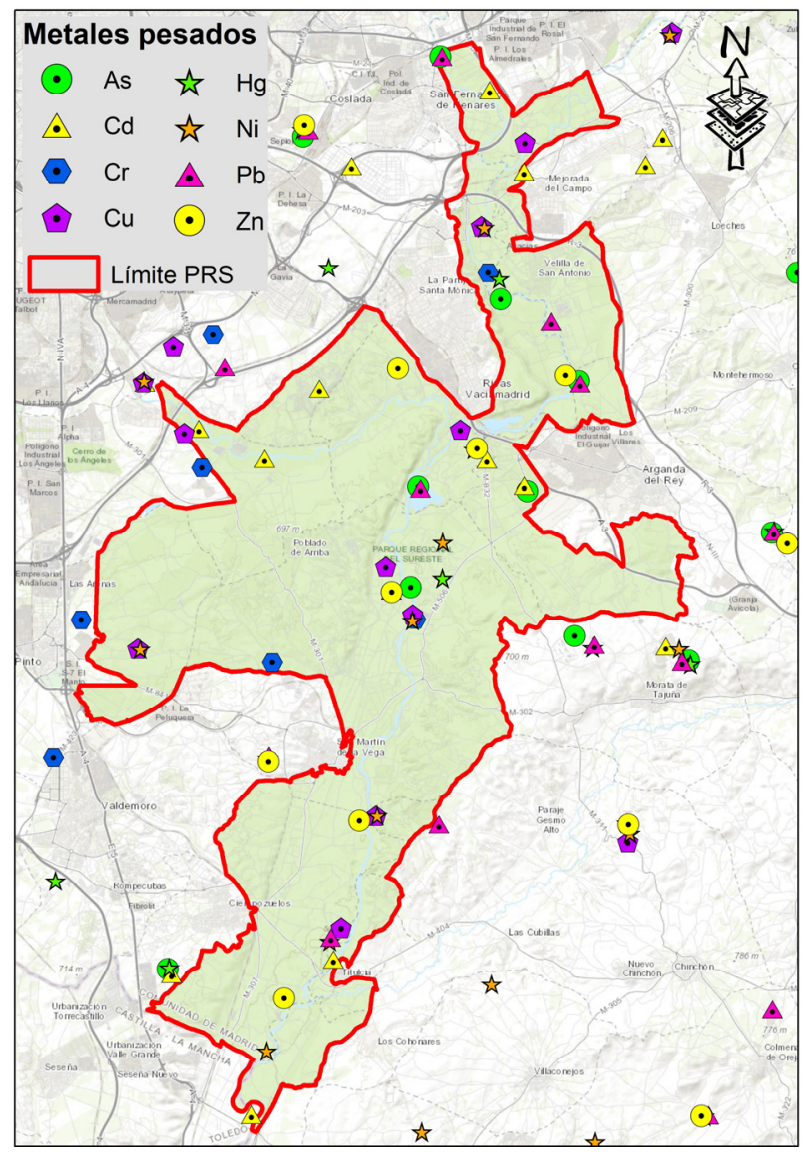

Figura 5: Espacialización de puntos con posible presencia de metales pesados a ser validados en campañas de campo. 
La selección de la CP debe tomar en cuenta tanto las cargas factoriales como los signos opuestos, dentro de la matriz de vectores propios, principalmente a partir de aquellas bandas de mayor contraste espectral. En el supuesto caso de que los resultados presenten un sin número de falsos positivos se debería optar por otra $\mathrm{CP}$, en caso de seguir con este inconveniente, se debería elegir otras bandas reflectivas y absortivas de partida.

El bajo contenido de bandas que presenta la imagen Sentinel-2 es una limitación para poder trabajar con longitudes de onda específicas, donde se evidencian los mayores contrastes espectrales tanto de absortividad como de reflectividad. A pesar de esto, los resultados obtenidos de los minerales bentonita y sepiolita concuerdan con la cartografía de contraste. El escaso número de bandas podría ser una fuente de error, debido a que los metales pesados tenderían a traslaparse con longitudes de onda de absorción de óxidos de hierro, materia orgánica, agua y minerales arcillosos.

Se evidencia una similitud espacial por parte de algunos metales, por ejemplo, As, Cd y Pb se encuentran en los mismos objetivos o superficies, al igual que $\mathrm{Ni}$ y $\mathrm{Cr}$. Todos estos puntos serán corroborados mediante medidas con espectroradiómetro, análisis químico de muestras e información de organismos rectores en la temática.

Otra de las acciones a corto plazo, es la aplicación de la metodología en tierras agro-forestales, para poder determinar posibles zonas afectadas por metales pesados, orientado a dar cumplimiento de la normativa de la Unión Europea en cuanto a niveles máximos de elementos traza.

El empleo de datos multifuente como las imágenes super o hiperespectrales permitirá afinar la metodología que se presenta en el artículo, gracias a su elevado número de bandas, teniendo la posibilidad de aislar los elementos con una mayor precisión.

Por último, se realizará una transferencia de tecnología, en donde las habilidades, conocimiento y herramientas adquiridas, serán trasladadas desde España a Ecuador, con un doble enfoque, por un lado, esto permitirá la intercalibración de algoritmos generados, y por otro, se tendrá la capacidad para poder detectar aquellas zonas afectadas por metales pesados.

\section{Agradecimientos}

La presente investigación tiene el apoyo de:

- Secretaría de Educación Superior, Ciencia, Tecnología e Innovación (SENESCYT), mediante la beca otorgada dentro del marco de la convocatoria abierta 2017 - componente general.

- Instituto Geográfico Militar del Ecuador (IGM), mediante la concesión del permiso respectivo para poder efectuar estudios regulares de posgrado en España.

- Tepro Consultores Agrícolas SL, mediante las prácticas doctorales realizadas a partir del 01/03/2021 hasta la actualidad.

\section{References}

ADRIANO, D., 2001. Trace elements in terrestrial environments: biogeochemistry, bioavailability, and risks of metals. Second edn. Verlag, New York, Berlin, Heidelberg: Springer.

ARELLANO, P., TANSEY, K., BALZTER, H. and BOYD, D.S., 2015. Detecting the effects of hydrocarbon pollution in the Amazon forest using hyperspectral satellite images. Environmental Pollution, 205, pp. 225-239.

BRAGADO, E., REJAS, J.G., MARCHAMALO, M. and BONATTI, J., 2009. Delimitación de zonas de alteración hidrotermal en la Cordillera Volcánica Central de Costa Rica mediante imágenes Landsat y del sensor simulador MASTER, S. MONTESINOS and L. FERNÁNDEZ, eds. In: XIII Congreso de la Asociación Española de Teledetección, September 23, 2009, pp. 265-268.

CASTILLEJO, D.G., 2019. Ecologistas piden investigar un vertido de lodos de aguas residuales en el Parque Regional del Sureste.

CM. 2019. Datos Abiertos de la Comunidad de Madrid.

CRÓSTA, A.P., DE SOUZA FILHO, C. R., AZEVEDO, F. and BRODIE, C., 2003. Targeting key alteration minerals in epithermal deposits in Patagonia, Argentina, using ASTER imagery and principal component analysis. International Journal of Remote Sensing, 24(21), pp. 4233-4240.

DAS, B., MANOHARA, K.K., MAHAJAN, G.R. and SAHOO, R.N., 2020. Spectroscopy based novel spectral indices, PCAand PLSR-coupled machine learning models for salinity stress phenotyping of rice. Spectrochimica Acta Part AMolecular and Biomolecular Spectroscopy, 229, pp. 1-13.

DAVIDS, C. and TYLER, A.N., 2003. Detecting contamination-induced tree stress within the Chernobyl exclusion zone. Remote Sensing of Environment, 85(1), pp. 30-38.

DURÃES, N., NOVO, L.A.B., CANDEIAS, C. and DA SILVA, E.F., 2017. Distribution, transport and fate of pollutants. Soil Pollution: From Monitoring to Remediation. Elsevier, pp. 29-57.

FONSECA, D., 2016, Nov 21. La espuma que amenaza el Jarama. El País. ISSN 1134-6582.

GALÁN, E. and ROMERO, A., 2008. Contaminación de suelos por metales pesados. Revista de la sociedad española de mineralogía, 10, pp. 48-60.

IGME. 1998. Cartografía geológica del Instituto Geológico y Minero de España. 
IGN. 2019. Centro Nacional de Información Geográfica del Instituto Geográfico Nacional.

IGN. 2011. Sistema de Información de Ocupación del Suelo de España.

JPL. 2018. ECOSTRESS Speclib de Jet Propulsion Laboratory. Search Library - ECOSTRESS Speclib (nasa.gov). Available: https://speclib.jpl.nasa.gov/library [5/12, 2021].

LONDOÑO-FRANCO L, LONDOÑO-MUÑOZ P, and MUÑOZ-GARCÍA F., 2016. Los riesgos de los metales pesados en la salud humana y animal. Biotecnología en el Sector Agropecuario y Agroindustrial, 14(2), pp. 145-53.

LOUGHLIN, W.P., 1991. Principal component analysis for alteration mapping. Photogrammetric Engineering \& Remote Sensing, 57(9), pp. 1163-1169.

MEZA, K., RUALES, B., MAIGUASHCA, J. and RIVADENEIRA, J.L., 2020. Caracterización espectral de estrés hídrico en el cultivo de pepino dulce (Solanum muricatum). Geoespacial, 17(1), pp. 14-24.

REJAS, J.G., MARTÍNEZ-FRÍAS, J., MARTÍNEZ, R., MARCHAMALO, M. and BONATTI, J., 2014. Spectral analysis of geological materials in the Central Volcanic Range of Costa Rica and its relationship to the remote detection of anomalies. Estudios Geologicos, 70(2).

ROSSO, P.H., PUSHNIK, J.C., LAY, M. and USTIN, S.L., 2005. Reflectance properties and physiological responses of Salicornia virginica to heavy metal and petroleum contamination. Environmental Pollution, 137(2), pp. 241-252.

SHIN, H., YU, J., WANG, L., JEONG, Y. and KIM, J., 2020. Spectral interference of heavy metal contamination on spectral signals of moisture content for heavy metal contaminated soils. IEEE Transactions on Geoscience and Remote Sensing, 58(4), pp. 2266-2275.

SIEGEL, F.R., 2002. Environmental geochemistry of potentially toxic metals. Springer.

SOCA-MUÑOZ, J., RODRIGUEZ-MACHADO, E., ADAY-DIAZ, O., HERNANDEZ-SANTANA, L. and OROZCOMORALES, R., 2020. Spectral signature of brown rust and orange rust in sugarcane. Revista Facultad De IngenieriaUniversidad De Antioquia, 96, pp. 9-20.

YAO, X., ZHU, Y., TIAN, Y., FENG, W. and CAO, W., 2010. Exploring hyperspectral bands and estimation indices for leaf nitrogen accumulation in wheat. International Journal of Applied Earth Observation and Geoinformation, 12(2), pp. 89100. 